\title{
Comparison of clinical outcomes according to the history of previous gastrectomy in patients undergoing pancreatoduodenectomy: a propensity score matching analysis
}

Jae Ri Kim, Wooil Kwon, Jihoon Chang, Jin-Young Jang, Sun-Whe Kim

Department of Surgery, Cancer Research Institute, Seoul National University College of Medicine, Seoul, Korea

https://doi.org/10.4174/astr.2020.98.4.177

Annals of Surgical Treatment and Research 2020;98(4):177-183

To the Editor:

Thank you for publishing our article titled "Comparison of clinical outcomes according to the history of previous gastrectomy in patients undergoing pancreatoduodenectomy: a propensity score matching analysis" in volume 98 of April 2020 issue. In the original publication, we found a mistake in IRB number. The mistake was not due to the editorial office of Annals of Surgical Treatment and Research. We attached a corrigendum along with this letter to request for correction of IRB number.

We want to correct IRB number in METHODS section of this article.

Your sincerely,

Sun-Whe Kim

Corrected IRB number

The study protocols were approved by the Institutional Review Board of Seoul National University Hospital (IRB No. H-1705-006851). 